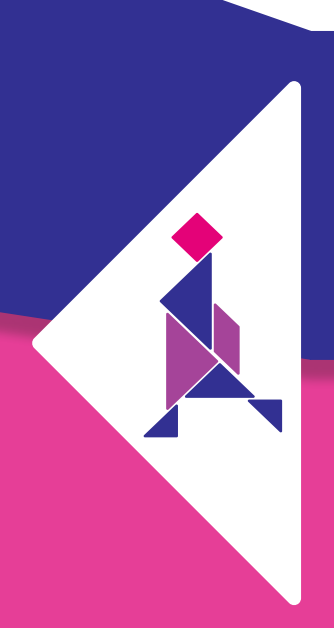

\title{
Efeito do exercício calistênico na dor e capacidade cardiorrespiratória de idosos com Doença Arterial Obstrutiva Periférica
}

\author{
Effect of calisthenic exercise in pain \\ and cardiorespiratory fitness of elderly \\ patients with Peripheral Arterial Disease
}

FisiSenectus . Unochapecó Ano 3, n. 2 - Jul/Dez. 2015 p. $47-58$

Lilian Marin. fisiolili@unochapeco.edu.br

Docente do curso de Fisioterapia da Universidade Comunitária da Região de Chapecó

(Unochapecó), Chapecó, SC- Brasil.

Alessandra Paula Salerno. alessandra_salerno@unochapeco.edu.br

Fisioterapeuta graduada em Fisioterapia pela Universidade Comunitária da Região de Chapecó (Unochapecó), Chapecó, SC- Brasil.

Flavia Variani. flaviavariani@unochapeco.edu.br

Fisioterapeuta graduada em Fisioterapia pela Universidade Comunitária da Região de Chapecó (Unochapecó), Chapecó, SC- Brasil.

\begin{abstract}
Resumo
Introdução: A Doença Arterial Obstrutiva Periférica (DAOP) é uma doença vascular que provoca diminuição ou obstrução dos vasos que conduzem o sangue. 0 tratamento fisioterapêutico é apontado como o primeiro procedimento a ser adotado no combate da DAOP e seus sintomas clínicos, uma vez pode ser utilizado em todos os indivíduos, pela sua facilidade de aplicabilidade. Objetivo: Verificar o efeito de um programa de exercício fisioterapêutico de curto prazo em idosos com DAOP. Materiais e métodos: Foi realizado um estudo descritivo do tipo relato de caso no qual participaram três idosos, estes foram submetidos à avaliação fisioterapêutica com a utilização da escala de claudicação de Edimburg (1983), escala de Borg, escala visual analógica do dor (EVA) e teste de caminhada de seis min, após serem submetidos a um protocolo de exercícios de dez intervenções consecutivas. Conclusão: Com a análise dos resultados pôde-se verificar que a intervenção fisioterapêutica precoce em indivíduos com DAOP há menos de três meses gerou boas respostas em curto período de tempo, o que evidenciou-se na variável EVA, a qual demonstrou melhores resultados, diminuindo de sete para zero. Na Distância Prevista obteve-se maiores resultados quando comparados à avaliação inicial e final. Também houve modificações significativas na PAS e FC, quando comparadas à avaliação final e inicial.
\end{abstract}

Palavras-chave:

Fisioterapia. Terapêutica. Doenças Cardiovasculares. 


\begin{abstract}
Peripheral Arterial Obstructive Disease (PAOD) is a vascular disease that causes reduction or blockage of blood vessels that lead. The physical therapy is touted as the first procedure to be adopted in fighting the PAD and its clinical symptoms, as can be applied to all individuals, for their ease of applicability. Objective: To determine the effect of a short-term physical therapy exercise program in elderly patients with PAD. Methods: A descriptive study of case report type was conducted in which three elderly, they underwent physical therapy assessment using the Edinburgh claudication scale (1983), Borg scale, visual analogue scale of pain (VAS) and 6 min walk test after undergoing a protocol of 10 consecutive interventions exercises Conclusion: With the analysis of the results can be seen that early physical therapy intervention in individuals with PAD for less than three months generated good responses in short time, was evidenced in EVA variable which showed better results, decreasing from 07 to 0 . The Distance Planned Obtiva up better results when compared to the initial and final evaluation. There were also significant changes in SBP and HR compared initial and final evaluation.
\end{abstract}

\title{
Keywords:
}

Physiotherapy. Therapeutics. Cardiovascular Diseases.

\section{Introdução}

$\infty \times \infty \times \infty \times \infty \times \infty \times \infty \times \infty \times \infty \times \infty \times \infty \times \infty \times \infty \times \infty)$

O envelhecimento é um processo pelo qual todos os indivíduos e organismos passam, é caracterizado pela diminuição gradativa das capacidades dos vários sistemas orgânicos em conseguir realizar suas funções de maneira eficaz. Essas alterações ocorrem em ritmo e momentos diferentes. Assim, o envelhecimento biológico normal leva a algumas implicações funcionais que podem levar o idoso à perda de autonomia e a uma consequentemente dependência de parentes e amigos $^{1}$.

No processo fisiológico do envelhecimento, o organismo sofre várias modificações bioquímicas, morfológicas e funcionais, sendo que essas mudanças são caracterizadas por atrofias, limitações físicas, perdas cognitivas, sintomas depressivos, diminuições no funcionamento de diversos órgãos e principalmente aparecimento de doenças crônicas ${ }^{2}$.

O aumento nos casos de doenças crônicas está diretamente relacionado com maior incapacidade funcional e esta vem sendo definida como grau de preservação do indivíduo na capacidade de realizar atividades básicas de vida diária (ABVDS) e atividades instrumentais de vida diária (AIVDS). Quando a capacidade funcional começa a diminuir as limitações começam a se destacar, causando implicações importantes para a família, comunidade e para o sistema de saúde, além de maior vulnerabilidade e dependência na velhice, contribuindo para a diminuição do bem-estar e da qualidade de vida ${ }^{3}$.

As doenças crônicas comumente encontradas em idosos são as de origem musculoesquelética, respiratórias, renais, endócrinas, distúrbios emocionais, cardiovasculares e circulatórias, destacando-se a Doença Arterial Obstrutiva Periférica $(D A O P)^{4}$.

A DAOP é uma doença vascular que afeta os sistemas arterial, venoso e linfático, provocando diminuição ou obstrução dos vasos que conduzem o sangue e prejudicando o fluxo normal. A primeira manifestação clínica da doença é a Claudicação Intermitente (CI), esta consiste em quadros de dor, cãibra, formigamento ou ardência com maior frequência na musculatura posterior da perna ${ }^{5}$.

Um dos fatores de risco para o desenvolvimento das doenças crônicas vasculares e que acaba predispondo a DAOP é a hipertensão arterial (pressão sistólica acima de 140 mmHg e diastólica maior que $90 \mathrm{mmHg}$ ). A presença desta acaba por acarretar maior risco a ataques cardíacos, além de ser um importante fator de determinante para 0 AVC. Outro fator de risco que vem crescendo é a presença de lipídios sanguíneos anormais, níveis de colesterol total e triglicerídeos aumentados, 
juntamente com baixos níveis de $\mathrm{HDL}$, os quais aumentam o risco de doenças coronarianas e AVC isquêmico².

Frente a esse cenário perturbador, esforços têm sido realizados na tentativa de encontrar soluções para a prevenção e o tratamento da DAOP6 ${ }^{6}$. A equipe multiprofissional tem importante função nesse contexto, uma vez que sua abordagem acontece de forma diferenciada, corrigindo a grande limitação no tratamento dos idosos, melhorando a adesão ao programa de atendimento e o controle da doença ${ }^{7}$.

Dentre os profissionais que compõem a equipe multiprofissional, o tratamento realizado pelo fisioterapeuta é apontado como um dos primeiros procedimentos a serem adotado no combate da DAOP e seus sintomas clínicos ${ }^{8}$. Os programas de exercícios fisioterapêuticos em indivíduos com DAOP proporcionam uma melhora da tolerância ao esforço físico, acompanhada de uma melhora da capacidade deambulatória, força muscular, flexão plantar, aumento da capilarização da musculatura da perna, velocidade de caminhada, capacidade de subir escadas e consequentemente uma melhora na qualidade de vida. Além disso, uma das vantagens importantes é a ausência de claudicação durante a execução dos exercícios, fatores esses que auxiliam na recuperação do paciente ${ }^{9}$.

Verifica-se que a maioria dos estudos são relacionadas à modalidade de treinamento aeróbio, resistidos ou associados. Estes possuem risco de viés, alguns são limitados e não padronizados em relação aos protocolos de exercícios no que diz: modalidade do exercício, tempo diário de intervenção, período de duração do tratamento e modo de progressão dos exercícios. Dessa forma, destaca-se a importância de avaliar o efeito de um programa de intervenção fisioterapêutica, com exercícios de baixa intensidade e curta duração (calistênicos) no âmbito da dor e da capacidade cardiorrespiratória em idosos com DAOP.

\section{Metodologia}

$\infty \times \infty \times \infty \times \infty \times \infty \times \infty \times \infty \times \infty \times \infty \times \infty \times \infty \times \infty \times \infty$

0 presente estudo é caracterizado como uma pesquisa quantitativa experimental. A população foi constituída por idosos do Município de Chapecó
- SC com diagnóstico DAOP há menos de três meses. Dentre os participantes dois eram do sexo masculino e um do sexo feminino, com faixa etária de 60 a 80 anos. Os critérios de inclusão foram: estar dentro da faixa etária preconizada, que deambulassem sem auxílio de terceiros, apresentassem arteriosclerose com claudicação reversível e grau subjetivo de claudicação II e III a pelo menos seis meses, ter recomendação do médico-cirurgião cardiovascular para realização de exercícios supervisionados e serem hipertensos medicalizados. Ressalta-se que a amostra não foi maior devido à baixa demanda médica e inadequação aos critérios de inclusão.

Os sujeitos participantes da pesquisa assinaram o termo de consentimento livre e esclarecido, após foram submetidos a uma avaliação fisioterapêutica e ao final de dez intervenções consecutivas, constituída por: dados pessoais, hábitos de vida, sinais vitais, aspectos relacionados ao sistema cardiovascular (teste de Homan e teste de Thompson) e avaliação da dor com a escala análogo visual da dor - EAV (consiste de uma linha reta, não numerada, na qual uma extremidade marca a ausência de dor e a outra a pior dor imaginável) foi utilizada na avaliação inicial e final com objetivo de mensurar a dor referida pelo paciente ${ }^{9}$.

Na sequência, os pacientes foram submetidos ao teste de caminhada de seis minutos o qual consistiu em caminhar em um corredor plano de vinte metros, com incentivo verbal. Os parâmetros de Pressão Arterial (PA), Frequência Cardíaca (FC), Saturação de Oxigênio (Sp02) e Índice de Percepção Esforço de Borg (IPE) foram verificados no início e ao fim do teste ${ }^{9}$. Após, aplicou-se o Questionário de Claudicação de Edimburgo (1992), com o intuito de avaliar a presença e grau de claudicação. Este possui seis questões, com um diagrama para marcação do local exato da dor, facilitando sua autoaplicação. 0 resultado será classificado como POSITIVO (presença de claudicação) caso as respostas às perguntas tenham sido $1=$ "sim", 2 "não", 3 = "sim", 5 = "geralmente desaparece em dez minutos ou menos" e 6 = "panturrilha" e/ou "coxa" e/ou "região glútea"(independentemente de terem sido assinalados outros lugares) ou NEGATIVO se assinalada qualquer combinação diferente da descrita acima. A questão quatro 
não é utilizada para definir a presença e sim a gravidade da claudicação: "Não" = Grau 1(menor gravidade); e "Sim" = Grau 2 (maior gravidade) ${ }^{10}$.

Em seguida os indivíduos foram submetidos a um protocolo de exercícios cinesioterapêuticos baseados na literatura ${ }^{11,12}$ e adaptados com enfoque para o sistema vascular, conforme Quadro 1. o programa de exercícios foi realizado todos os dias, durante dez dias, com duração de 60 minutos cada intervenção. Os sinais vitais (PA, $F C$, fr) e EVA foram verificados no início e ao final de cada intervenção. 0 protocolo de exercícios de baixa intensidade e curta duração proposto para o estudo com os idosos possui inúmeros benefícios, pois não carece de equipamentos exclusivos, é de fácil aplicabilidade e não envolve custos adicionais, além disso, apresenta resultados significativos quando realizados em âmbito domiciliar.

As coletas foram realizadas no período de outubro a novembro de 2015, no laboratório de semiologia localizado na Unochapecó; No que se refere às faltas ocorridas durante o processo de coleta de dados, cabe mencionar que todas foram repostas.

A análise dos dados foi realizada em Microsoft Excel versão 2010, na forma de estatística descritiva, e os valores numéricos foram expressos por média e desvio-padrão e apresentados na forma de gráficos e tabelas.

\section{Resultados}

$\infty \times \infty \times \infty \times \infty \times \infty \times \infty \times \infty \times \infty \times \infty \times \infty \times \infty \times \infty \times \infty \times \infty$

Dos três indivíduos que realizaram as dez intervenções fisioterapêuticas, obteve-se uma média de idade de 73,66 $( \pm 5,5)$ anos. Quanto aos hábitos de vida, somente um dos entrevistados é tabagista, sendo os três sedentários e não faziam uso de bebidas alcoólicas. Com relação ao diagnóstico médico, todos apresentavam a DAOP associada à hipertensão arterial, sendo que apenas um apresentou Diabetes Mellitus. Na avaliação da claudicação, realizada por meio do questionário de claudicação de Edimburgo, todos os indivíduos apresentaram resultado positivo para claudicação, sendo que dois apresentaram grau 2, ou seja, maior gravidade, enquanto um participante apresenta grau 1, o que corresponde a menor gravidade.
Quanto à variável antropométrica, o Índice de Massa Corporal (IMC), dois dos entrevistados classificaram-se como pré-obesos, com valores entre 25 e 29,5, e um deles classifica-se como peso normal, com valores entre 18,5 e 24,9 . Os valores de IMC acima do considerado normal predispõem 0 aparecimento das doenças cardiovasculares ${ }^{13}$.

A pressão arterial sistêmica foi avaliada e monitorada ao longo das dez intervenções. Pôde-se observar que os valores encontravamse elevados quando comparados aos valores de normalidade (pressão sistólica abaixo de 120 $\mathrm{mmHg}$ e diastólica menores que $80 \mathrm{mmHg}$ ). Como observado no gráfico de evolução 1 , os valores de pressão arterial sistólica inicial elevaram-se no terceiro atendimento e em seguida sofreram um decréscimo no decorrer dos dez atendimentos. Já os valores de pressão arterial diastólica inicial e final, bem como a sistólica final, não sofreram alteração (Gráfico $\mathbf{1}$ ).

Referente à $F C$, como é possível observar no Gráfico 2, nota-se que houve um decréscimo tanto na inicial quanto na final no decorrer das dez intervenções. Na primeira intervenção a FC inicial obteve uma média de 81 btm e ao final uma média de 74 btm, já a FC final também sofreu decréscimo, passando de $77 \mathrm{btm}$ para $68 \mathrm{btm}$.

A média da FR inicial foi atribuída com 18 incursões respiratórias por minuto (irpm), já a FR final obteve uma média de 17 irpm. Dessa forma, podemos constatar que esta não sofreu alteração significativa no decorrer de dez atendimentos. $\mathrm{Na}$ mensuração da oximetria, todos os participantes apresentaram uma média inicial e final de Sat02 95\% (Tabela $\mathbf{1}$ ).

Quanto à dor relatada em MMII e avaliada por meio da EVA, pôde-se observar no gráfico um decréscimo diário ao final das dez intervenções. $\mathrm{Na}$ avaliação inicial esta obteve uma média de 7 e ao final das dez intervenções classificou-se em 1 , já na avaliação final iniciou com 4 e passou para 0.

0 teste de caminhada de seis minutos foi realizado na avaliação e na reavaliação. É um teste usado para avaliar a resposta de um indivíduo ao exercício e propicia uma análise global dos sistemas respiratório, cardíaco e metabólico. Como visualizado na tabela, todos os pacientes obtiveram uma melhora na distância percorrida 
quando comparados ao resultado da reavaliação, com destaque para o participante 3, que percorreu uma distância mais significativa que os demais (Tabela 2).

\section{Discussão}

$\infty \times \infty \times \infty \times \infty \times \infty \times \infty \times \infty \times \infty \times \infty \times \infty \times \infty \times \infty \times \infty)$

De todas as variáveis avaliadas ressaltase que estas demonstram melhores resultados comparando à avaliação inicial e à reavaliação após as dez intervenções: EVA, PAS, FC e distância percorrida em teste de caminhada dos seis minutos.

Nessa perspectiva, a presença de dor, PAS e FC no idoso constituem um problema de relevância no contexto atual social e de saúde, principalmente quando estas influenciam na limitação da capacidade funcional. 0 idoso que mantém sua independência e autodeterminação deve ser considerado saudável, ainda que apresente uma ou mais doenças crônicas ${ }^{14}$.

Segundo o estudo realizado por Teixeira e Figueiró ${ }^{15}$, quando temos como sintoma a dor, utensílios de autorrelato são os mais utilizados para qualifica-la e verificar o impacto que esta exerce na vida dos indivíduos. A dor é caracterizada, principalmente, por história natural prolongada, fatores de risco complexos e múltiplos, interação de fatores etiológicos de causa ou especificidade desconhecida ${ }^{16}$. Esta pode dificultar a realização de AVDs, ocasionando dificuldade relativa na realização da marcha, instabilidade funcional e alto risco de quedas ${ }^{17}$.

Em estudo, Oliveira, Fernandes e Daher ${ }^{18}$ objetivaram investigar o efeito de um programa de exercícios físicos sobre a dor crônica inespecífica. Participaram 29 indivíduos que aderiram a um programa de exercícios resistidos, de alongamentos e aeróbicos. Estes foram avaliados antes e após três meses por meio do questionário EVA. Constataram então que a aplicação de um programa de exercícios aeróbicos, de fortalecimento e alongamentos apresentou melhora efetiva no limiar de dor em paciente com dor crônica inespecífica.
Tais resultados se assemelham com aqueles encontrados neste estudo, no qual se constatou significância entre os momentos antes e após as dez intervenções fisioterapêuticas na variável atribuída à dor segundo a Escala Analógica Visual da dor (EVA).

Já conforme as alterações nos valores pressóricos, o exercício físico promove redução da pressão arterial sistólica por diminuição no débito cardíaco que está associada ao decréscimo da frequência cardíaca ${ }^{19}$. A queda na resistência vascular sistêmica é outro mecanismo alternativo proposto para explicar a queda na pressão arterial pós-exercício. Uma redução significativa nos níveis pressóricos é conseguida com treinamento de baixa intensidade, este diminui a pressão arterial porque provoca redução no débito cardíaco, o que pode ser explicado pela diminuição na frequência cardíaca de repouso e diminuição do tônus simpático no coração ${ }^{20}$.

Em estudo, Ishikawa et al. ${ }^{21}$ analisaram 109 indivíduos hipertensos nos estágios I e II, que realizaram treinamento leve por oito semanas, em academias. Constataram que houve redução nos níveis pressóricos dos indivíduos.

Em outro estudo Graves e Franklin ${ }^{22}$ avaliaram o comportamento das pressões sistólica (PAS), diastólica (PAD) e média (PAM) após uma sessão de exercício resistido e de exercício aeróbico. A pressão arterial de 12 adultos hipertensos sedentários foi medida em repouso e durante $30 \mathrm{~min}$. após caminhada de $20 \mathrm{~min}$. em esteira elétrica, com sensação subjetiva de esforço moderada e após duas séries de 15 repetições com a mesma intensidade subjetiva nos exercícios: voador peitoral, leg-press horizontal, puxada pela frente no pulley alto, extensão de joelhos e flexão de joelhos. Os resultados identificaram redução significativa da PAS após o exercício aeróbio durante todo o período de monitorização pósesforço.

Podemos então igualar a eficácia da realização de exercícios resistidos, aeróbicos e de baixa intensidade com as mudanças pressóricas que foram observadas após dez intervenções de exercícios de baixa intensidade, comprovando sua eficiência. 
Crowther $^{23}$ define exercício físico como uma forma de atividade física planejada, repetitiva, que procura aumentar a resistência física e as habilidades motoras. No treinamento aeróbio o exercício depende da intensidade, duração e frequência, e o resultado final é uma adaptação cardiovascular que se evidência na resistência à fadiga. Na realização do exercício a frequência de despolarização do nódulo sinoatrial, do débito cardíaco, da frequência cardíaca e da força das fibras miocárdicas aumentam, ocorre uma redução na resistência do leito vascular arterial do músculo em trabalho e diminuição da resistência vascular periférica ${ }^{20}$.

Já no treinamento resistido os três elementos que desempenham função muscular força, potência e resistência à fadiga podem ser modificados por meio das variáveis intensidades, frequência e duração do exercício. A maioria dos programas de exercício busca manter o equilíbrio desses três fatores, proporcionando benefícios como o aumento do desempenho muscular e da força, a diminuição da sobrecarga articular, a redução das lesões em tecidos moles e a melhora do equilíbrio ${ }^{23}$.

Buscando investigar os efeitos do exercício resistido e aeróbio sobre a frequência cardíaca, Bornardi, Souza e Moraes ${ }^{24}$ aplicaram duas sessões de exercício resistido e aeróbio em 25 indivíduos sedentários e normotensos. Os exercícios resistidos foram realizados sob forma de circuito com pesos, com intensidade de $40 \%$ da força máxima individual, e os exercícios aeróbicos em ciclo ergômetro, com intensidade entre $60 \%$ e $70 \%$ da FC máxima alcançada no teste ergométrico. Os autores constataram que os exercícios resistidos provocaram elevação discreta, porém significativa de $\mathrm{FC}$ de 24 h na vigília e elevação da pressão arterial no sono, enquanto o exercício aeróbio promoveu redução significativa das pressões sem alterações de frequência cardíaca.

Pode-se evidenciar que o treino resistido e aeróbico promove oscilação na frequência cardíaca, tal oscilação pode ser observada também em exercícios de baixa intensidade e curta duração, estes acabam acarretando mudanças ao longo prazo, juntamente com alterações na pressão arterial e, consequentemente, no débito cardíaco, como demonstra o presente estudo.

Com ralação à capacidade cardiorrespiratória avaliada por meio do teste de caminhada de seis minutos, Fernandes e Pereira ${ }^{25}$ colocam que esse teste está sendo muito utilizado para estimar o desempenho funcional dos indivíduos de diferentes faixas etárias, pois possui boa resposta e requer mínimos recursos para sua execução.

Os autores Langoni e colaboradores ${ }^{26}$ realizaram um estudo com 33 idosos que foram qualificados segundo o Índice de Barthel como idosos independentes e semi- independentes, capazes de executar o teste de caminhada de seis minutos. Os idosos foram classificados como sedentários e com idades entre 60 a 80 anos, do sexo feminino e masculino, e os dados vitais eram mensurados ao início e ao término do teste, sendo que a média da distância máxima percorrida pelos participantes foi de 250 metros, muito abaixo da média esperada, que era prevista para 450 metros. Como resultado e conclusão do estudo foi constatado que não houve uma diferença considerada significante na comparação das médias em ambos os sexos, tanto no que diz respeito à distância percorrida quanto à prevista.

Em contrapartida, o nosso estudo não condiz com o estudo realizado por Langoni e colaboradores $^{26}$, pois se observou que o exercício tem função essencial no condicionamento aeróbico e no ganho muscular (força, potência e resistência), aumentandoassimo condicionamento cardiorrespiratório ${ }^{25}$

Quanto às variáveis menos expressivas, a presença do sedentarismo mostrou-se presente em todos os entrevistados, sendo possível associar o sedentarismo à DAOP, uma vez que a redução na atividade física pode ser reflexo do comprometimento funcional dos membros inferiores ocasionados pela dor e desconforto na região da panturrilha durante a deambulação ${ }^{26}$.

É importante ressaltar também a relação entre a doença crônica e à DAOP, nos idosos devido a uma combinação de fatores sociais, culturais, ambientais e comportamentais, onde os portadores apresentam características como o tabagismo, a hipertensão arterial, a obesidade e 0 Diabete Melitus ${ }^{27}$. Análises preliminares 
dos idosos longevos apontaram a obesidade como um dos fatores de risco cardiovascular mais prevalente. Taxas elevadas de sobrepeso estão sendo observadas no mundo inteiro. Uma das causas desse fenômeno está relacionada ao aumento na ingestão de gordura e açúcar e à diminuição do consumo de fibras e carboidratos ${ }^{28}$.

Nieman ${ }^{29}$ coloca que a gordura corporal quase dobra entre os 20 e os 65 anos de idade. Durante a meia-idade, a gordura corporal extra é concentrada em torno do abdômen e do tronco, o que é prejudicial à saúde em longo prazo. Essa alteração acontece exatamente na mesma época que as massas musculares e ósseas diminuem, fazendo com que ocorra uma queda na taxa metabólica, aumentando o trabalho cardíaco e vascular.

Sendo assim, a prática do exercício físico tende a diminuir os fatores de risco, além proporcionar uma melhora na capacidade funcional e na redução nas limitações periféricas fazendo com que o individuo obtenha mais autonomia e qualidade de vida.

Dessa maneira, ressalta-se a importância do exercício físico para o idoso, visto que, com o declínio das capacidades físicas e as mudanças fisiológicas advindas do processo do envelhecer, geram-se danos funcionais que colaboram para a dependência física.

A prescrição de exercícios é de extrema importância e de grande valia e necessita estar direcionada às alterações provocadas pelo envelhecimento. Os idosos tendem a corresponder de forma positiva, contudo, para que se obtenha um resultado gradativo e mais acelerado, os exercícios devem ser praticados de maneira regular e por meio de estímulos. É necessário, ainda, averiguar a legítima probabilidade de o treinamento refletir sobre os elementos da capacidade física do idoso e em que dimensão isso acontece, a fim de que oferecer objetivos realizáveis. A execução das atividades físicas realizadas de forma apropriada na terceira idade proporciona uma melhora no desempenho nas atividades e cuidados do dia a dia

\section{Considerações finais}

$\infty \times \infty \times \infty \times \infty \times \infty \times \infty \times \infty \times \infty \times \infty \times \infty \times \infty \times \infty)$

Pode-se concluir que as intervenções fisioterapêuticas precoces (há menos de três meses) em indivíduos com DAOP têm boas respostas em curto período de tempo, principalmente na variável EVA, a qual demonstrou melhores resultados, diminuindo de sete para zero. As intervenções também trazem a melhora no condicionamento cardíaco e muscular, devido à capacidade de adaptação do sistemas respiratório.

Evidenciou-se, também, que exercícios de baixa intensidade e curto período (calistênicos) melhoraram o desempenho físico da pessoa idosa e sua manutenção promove ou preserva o desempenho funcional. Ressalta-se que o protocolo proposto, por ser de fácil execução, pode ser realizado no ambiente domiciliar, atuando diretamente na melhora da execução das AVDs.

Sugere-se que exercícios de força e flexibilidade façam parte da prescrição fisioterapêutica em pacientes portadores de DAOP, pois são fundamentais para o melhor desempenho das atividades da vida diária, possibilitando aos pacientes maior autonomia.

Quanto às variáveis analisadas, a prevalência de PAS e sedentarismo trouxe a preocupação e a necessidade de uma atuação multiprofissional, possibilitando uma visão global do indivíduo, como na avaliação da necessidade de terapia medicamentosa nesses pacientes.

Os dados do presente estudo poderiam, ainda, ser mais expressivos se a amostra fosse maior, bem como o número de intervenções, porém percebe-se a importância da atividade física diária. Sugere-se outros estudos que comparem com grupo controle, separando gênero masculino e feminino e outras populações.

\section{Referências}

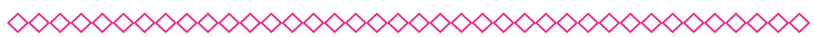

1. Fontes MA, Moreira OC, Gonzaga BT, Segheto $W$, Oliveira CE. Treinamento de força para terceira idade. EFDeportes.com. Buenos Aires. 2010; 140 (1).

2. Netto M, Brito F. Urgências em geriatria: epidemiologia, fisiopatologia, quadro clínico, conduta terapêutica. São Paulo: Atheneu. 2001. $476 \mathrm{p}$. 
3. Cesse EAP. Epidemiologia e determinantes sociais das doenças crônicas não transmissíveis no Brasil [dissertação]. Recife: Centro de Pesquisas Aggeu Magalhães, Fundação Oswaldo Cruz; 2007.

4. Pereira FM, Besse M. Fatores associados à independência funcional de idosos residentes em instituição de longa permanência. Acta Fisiátr. 2011; 18(2):66-70.

5. Carvalho T. Diretriz de reabilitação cardiopulmonar e metabólica: aspectos práticos e responsabilidades. Arquivos Brasileiros de Cardiologia. 2006 Jan; 86 (1).

6. Soares MM, Silva LOL, Dias CA, Rodrigues SM, Machado CJ. Adesão do idoso ao tratamento da hipertensão arterial sistêmica: revisão integrativa. Cogitare Enferm. 2012; 17(1): 144-50.

7. Júnior DP, Amaral RT, Veiga EV, Cárnio EC, Nogueira MS, Pelá IR. A farmacoterapia no idoso: revisão sobre a abordagem multiprofissional no controle da hipertensão arterial sistêmica. Rev. Latino-Am. Enfermagem, Ribeirão Preto. 2006; 14(3): 435-41.

8. Ritti-Dias RM, Wolosker N, Forjaz CLM, Carvalho CRF, Cucato GG, Leão PP et al. Strength training increases walking tolerance in intermittent claudication patients: randomized trial. J Vasc Surg, 51 (2010), pp. 89-95.

9. Câmara LC, Santarém JM, Wolosker N, DiasRitti RM. Exercícios resistidos terapêuticos para indivíduos com doença arterial obstrutiva periférica: evidências para a prescrição. J. vasc. bras. 2007 Set; 6( 3 ): 246-256.

10. O'sullivan S, Schmitz T. Fisioterapia: avaliação e tratamento. São Paulo: Manole; 2004. 1152 p.

10. Makdisse M, Nascimento NR, Chagas ACP, Brasil D, Borges JL, Oliveira A et al. Versão em português, adaptação transcultural e validação do Questionário de Claudicação de Edimburgo. Arq. Bras. Cardiol. 2007 Mai; 88(5): 501-506.
11. Ritti-Dias RMR, Salvador EP, Wolosker N, Marucci MFN. Novas tendências no tratamento de indivíduos com claudicação intermitente por meio do exercício físico. Rev Bras Cienc Mov.2006; 14:111-5.

12. Locatelli EC, Pelizzari S, Scapini KB, Leguisamo CP, Silva AB. Exercícios físicos na doença arterial obstrutiva periférica. J Vasc Bras 2009 set; 8(3):247-254.

13. Soares MM, Silva LOL, Dias CA, Rodrigues SM, Machado CJ. Adesão do idoso ao tratamento da hipertensão arterial sistêmica: revisão integrativa. Cogitare Enferm. 2012 Jan/Mar; 17(1): 144-50.

14. Kohlbeck $D$, Lenardt $M$, Michel $T$, Setoguchi $L$, Grden C, Oliveira E. Fatores contributivos para a independência funcional de idosos longevos. Rev Esc Enferm USP. 2015; 49(1): 89-95.

15. Teixeira MJ, Figueiró JAB, Marcon RM, Rocha RO. Dor: Epidemiologia, Fisiopatologia, Avaliação, Dolorosas e Tratamento. São Paulo: Grupo Editorial Moreira Júnior: 2001. p.1-7.

16. Oliveira-Campos M, Rodrigues Neto, JF, Silveira MF, Neves DMR, Vilhena JM, Oliveira JF et al. Impacto dos fatores de risco para doenças crônicas não transmissíveis na qualidade de vida. Ciênc. saúde coletiva. 2013 Mar; 18( 3 ): 873-882.

17. Felipe LK, Zimmermann A. Doenças crônicas degenerativas em idosos: dados fisioterapêuticos. Rev Bras Promoç Saúde 2011;24(3):221-7.

18. Oliveira MAR, Fernandes RSC, Dahe SS. Impacto do exercício na dor crônica. Rev Bras Med. Esporte. 2014 Mai/Jun; 20 (3).

19. Negrão CE, Rondon MUPB, Kuniyosh FHS, Lima EG. Aspectos do treinamento físico na prevenção da hipertensão arterial. Revista Hipertensão. 2001; 4. Disponível em URL: http//www.sbh.org. br/revista/2001_2001_V4. Acesso em 11 maio 2003.

20. Baroni BM, Bruscatto CA, Rech RR, Trentin $L$, Brum LR. Prevalência de alterações posturais em praticantes de musculação. Fisioter. mov. (Impr.). 2010 Mar; 23( 1 ): 129-139. 
21. Ishikawa $K$, Ohta $T$, Zhang J, Hashimoto $\mathrm{S}$, Tanaka $\mathrm{H}$. Influence of age and gender on exercise training -induced blood pressure reduction in systemic hypertension. Am J Cardiol. 1999 Jul 15; 84 (2):192-6.

22. Graves EJ, Franklin BA. Treinamento resistido na saúde e reabilitação. Rio de Janeiro: Revinter; c 2006. 420 p.

23. Crowther RG, Spinks WL, Leicht AS, Sangla $\mathrm{K}$, Quigley F, Golledge J. Effects of a longterm exercise program on lower limb mobility, physiological responses, walking performance, and physical activity levels in patients with peripheral arterial disease. J Vasc Surg. 2008;47:303-9

24. Bonardi G, Souza VM, Moraes JFD. Incapacidade funcional e idosos: um desafio para os profissionais de saúde. Sci. Med. 2007; 17(3): 138-139.

25. Fernandes PM, Pereira NH, Santos ACBC, Soares MESM. Teste de Caminhada de Seis Minutos: avaliação da capacidade funcional de indivíduos sedentários. Rev Bras Cardiol. 2012; 25(1): 185-191.

26. Langoni C, Borsatto A, Valmorbida L, Resende T. Teste de caminhada de seis minutos em idosos de uma instituição de longa permanência: valores, aplicabilidade e correlações. RBCEH, Passo Fundo. 2013 set./dez; 10 (3) 285:295.

27. Curb JD, Masaki K, Rodriguez BL, Abbott RD, Burchfiel CM, Chen R, et al. Peripheral Arterial Disease and Cardiovascular Risk Factors in the Elderly. The Honolulu Heart Program. Arterioscler Thromb Vasc Biol. 1996; 16: 1495-500.

28. Da Cruz IBM, Almeida MSC, Schwanke CHA, Moriguchi EH. Prevalência de obesidade em idosos longevos e sua associação com fatores de risco e morbidades cardiovasculares. Rev. Assoc. Med. Bras. 2004 Abr; 50 (2): 172-177.

29. Nieman, D. Exercício e saúde. São Paulo: Manole; 2001. 317 p. 


\section{Anexos}

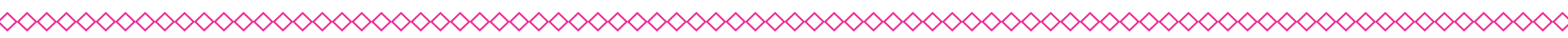

Quadro 1: Protocolo de Exercícios Cinesioterapêuticos

\begin{tabular}{|c|c|}
\hline Exercício & Descrição \\
\hline 01 & $\begin{array}{l}\text { Em pé, braços apoiados em uma cadeira, o paciente realizará flexão } \\
\text { plantar, elevando ambos os calcâneos durante } 1 \text { minuto e após mais } 1 \\
\text { minuto alternadamente. }\end{array}$ \\
\hline 02 & $\begin{array}{l}\text { Em pé, o paciente realizará uma caminhada apoiando somente o } \\
\text { antepé no solo e com calcâneos elevados por aproximadamente } 1 \\
\text { minuto. }\end{array}$ \\
\hline 03 & $\begin{array}{l}\text { Em decúbito dorsal, ao longo do corpo, flexão de quadril e joelhos, } \\
\text { pés apoiados no solo, realizará extensão de joelhos alternadamente. } \\
5 \text { vezes de } 15 \text {. }\end{array}$ \\
\hline 04 & $\begin{array}{l}\text { Em decúbito ventral, ombros em flexão de } 140^{\circ} \text {, cotovelos flexionados } \\
\text { medialmente, cabeça entre os braços, quadril neutro e joelhos } \\
\text { flexionados a } 90^{\circ} \text {, irá realizar flexão, extensão e circundução de } \\
\text { tornozelo. } 2 \text { min. }\end{array}$ \\
\hline 05 & $\begin{array}{l}\text { Em decúbito ventral, ombros em flexão de } 140^{\circ} \text {, cotovelos flexionados } \\
\text { medialmente, cabeça apoiada no dorso da mão, membros inferiores } \\
\text { em extensão apoiados no solo, paciente realizará flexão-extensão de } \\
\text { joelhos alternadamente. Inicialmente } 4 \text { vezes de } 15 \text { repetições. }\end{array}$ \\
\hline 06 & $\begin{array}{l}\text { Em decúbito dorsal, braços ao longo do corpo, membros inferiores } \\
\text { apoiados no solo. Paciente irá flexionar quadril e joelho, levando } \\
\text { o joelho em direção ao tórax os membros superiores estarão em } \\
\text { flexão de ombro } 90 \text { graus e cotovelos estendidos e acompanharão o } \\
\text { movimento do tronco. } 15 \text { vezes cada membro inferior. }\end{array}$ \\
\hline 07 & $\begin{array}{l}\text { Em decúbito dorsal com o quadril e joelhos flexionados, e os pés } \\
\text { apoiados ao solo, será realizado alternadamente movimento de flexão } \\
\text { de quadril em direção ao abdômen, será realizado durante } 1 \text { minuto, } \\
\text { pausa de } 15 \text { a } 20 \text { segundos e posteriormente mais } 1 \text { minuto. }\end{array}$ \\
\hline 08 & $\begin{array}{l}\text { Em decúbito ventral braços ao longo do corpo, quadris, joelho e } \\
\text { tornozelo neutro. Será posicionada uma almofada entre os tornozelos } \\
\text { do paciente, ele por sua vez terá que apertar a almofada fazendo uma } \\
\text { flexão com os dedos dos pés. } 5 \text { vezes por } 30 \text { segundos. }\end{array}$ \\
\hline
\end{tabular}




\section{Gráfico 1: Comportamento da Pressão Arterial Sistólica e Diastólica}

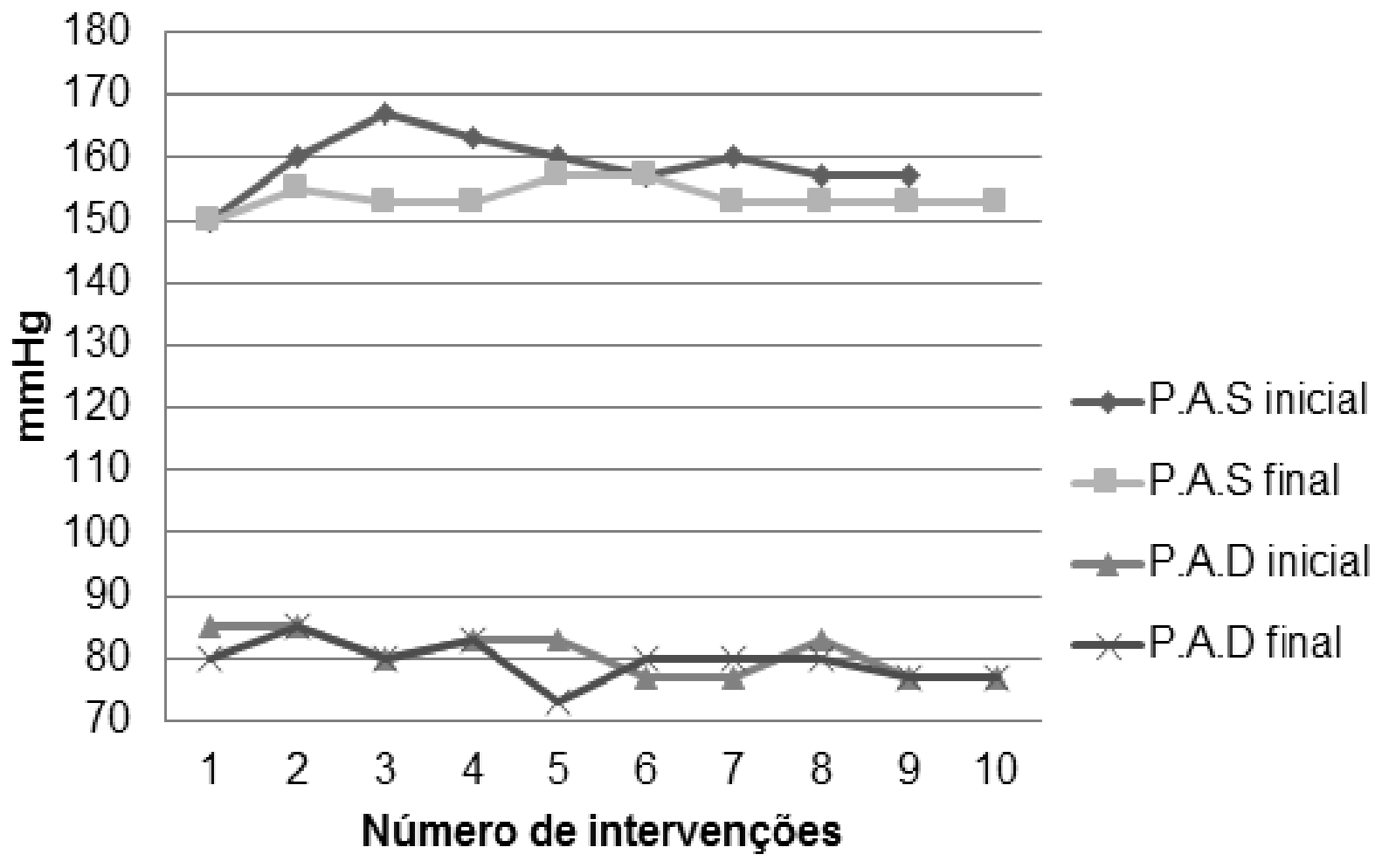

Fonte: Dados da Pesquisa.

Gráfico 2: Comportamento da Frequência Cardíaca

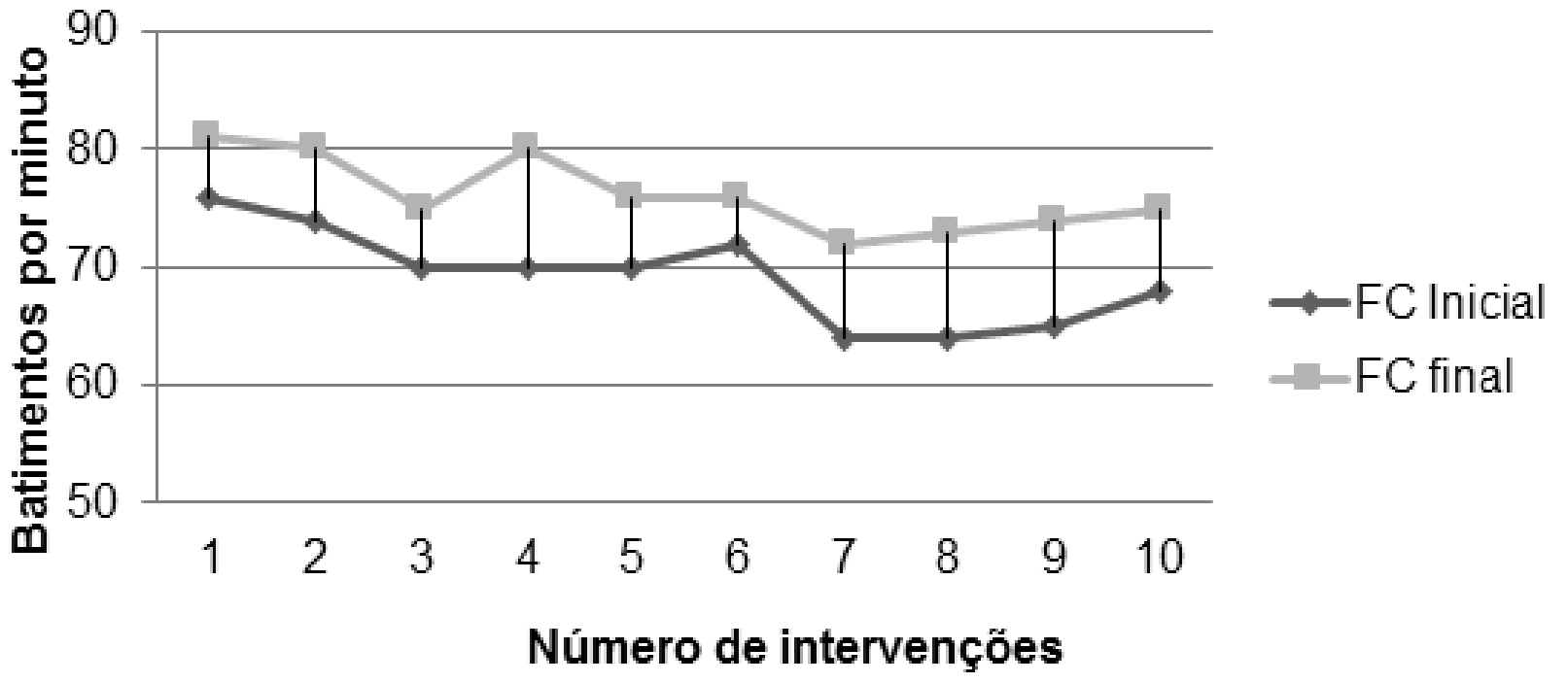

Fonte: Dados da Pesquisa. 
Tabela 1: Valores médios de frequência respiratória e oximetria

\begin{tabular}{|c|c|c|}
\hline & \multicolumn{1}{|c|}{ Inicial } & Final \\
\hline \multicolumn{2}{|c|}{ Frequência Respiratória } & \multicolumn{1}{c|}{$18( \pm 4)$} \\
\hline Sat02\% & $95( \pm 8)$ & $95( \pm 6)$ \\
\hline
\end{tabular}

Fonte: Dados da Pesquisa.

\section{Gráfico 3: Comportamento dor}

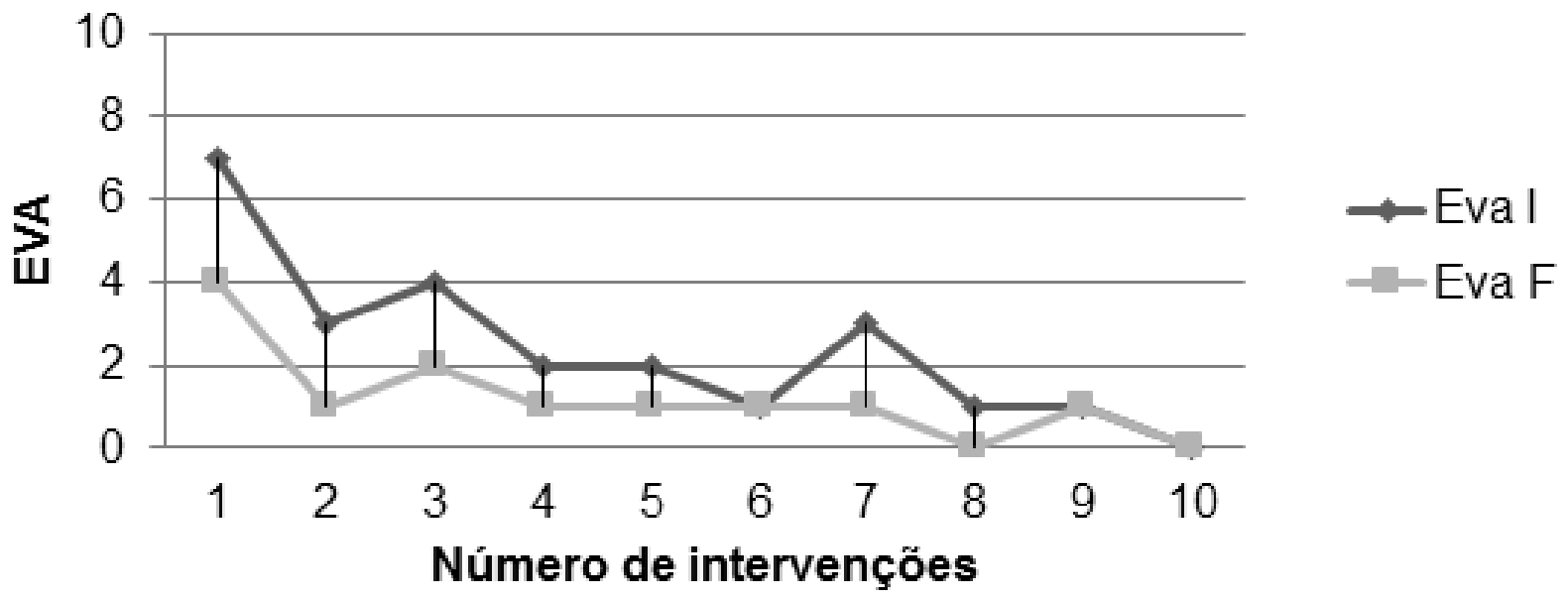

Fonte: Dados da Pesquisa.

(clique para voltar ao texto)

Tabela 2: Distância inicial e final analisada pelo teste caminhada 6 min.

\begin{tabular}{lccc} 
& Distância Prevista* & Distância Inicial* & Distância final* $^{*}$ Paciente 1 \\
Paciente 2 & 473,65 & 240 & 340 \\
Paciente 3 & 369,70 & 285 & 362 \\
& 444,7 & 250 & 421 \\
\hline
\end{tabular}

Fonte: Dados da Pesquisa.

Legenda: *Valores expressos em metros 